\title{
Conversion Disorder after Surgery in a Brain Tumour Patient
}

\author{
Kathleen Joy Khu, Sherese Ali, Mark Bernstein
}

Can. J. Neurol. Sci. 2010; 37: 293-295

We describe a case of conversion disorder in a young lady who had just been operated on for a glioma.

\section{Case Report}

A 31-year-old lady presented two years ago with seizures manifesting as jerking of her right arm and leg. Neurological examination revealed no focal deficits. Brain imaging revealed the presence of a small lesion in her left mesial posterior frontal lobe, just anterior to the motor strip (Figure 1). The appearance was compatible with a low grade glioma. There was no perilesional edema, enhancement, or mass effect. She was treated with anti-epileptic medications with good control of her seizures. The brain tumour was managed conservatively with regular clinical and imaging follow-up.

The patient remained well and asymptomatic, but her most recent magnetic resonance imaging (MRI) showed an increase in the size of the tumour (Figure 2). Awake craniotomy with cortical mapping for tumour excision was recommended and performed. Mapping over the motor cortex was positive, in that the right leg became weak when that area of cortex was stimulated. Corticotomy was made anterior to the motor cortex and aggressive subtotal resection of the tumour was achieved leaving behind a small cuff posteriorly, adjacent to the motor strip. As is our usual protocol, the patient was sedated after the mapping was performed, precluding continuous testing during tumour resection.

Immediately post-operatively, the patient could not move her right leg. Motor examination was $5 / 5$ for all limbs except for the right lower extremity which was $0 / 5$ in all muscle groups. There was no sensory deficit. The patient had hyperreflexia bilaterally but no Babinski. She was noted to have a positive Hoover's sign, with palpable downward force of the right leg while raising the unaffected left limb. The patient's pattern of leg weakness exhibited some inconsistencies: despite a motor strength of $0 / 5$, she was able to bear weight on her right leg and walk with support within two days. In addition, throughout the patient's hospital stay, she showed little concern about and seemed to be indifferent to her condition.

Various investigations were carried out. Computed tomogram four hours after surgery and MRI 40 hours post-operatively both revealed the resection cavity in the left posterior frontal area anterior to the precentral gyrus, and a small amount of residual tumour between the surgical site and the motor strip (Figure 3). There was no hemorrhage, increase in the perilesional edema, or mass effect. Neurophysiologic studies such as nerve conduction velocity, electromyography, and motor evoked potentials done two weeks after surgery confirmed no organic brain pathology.

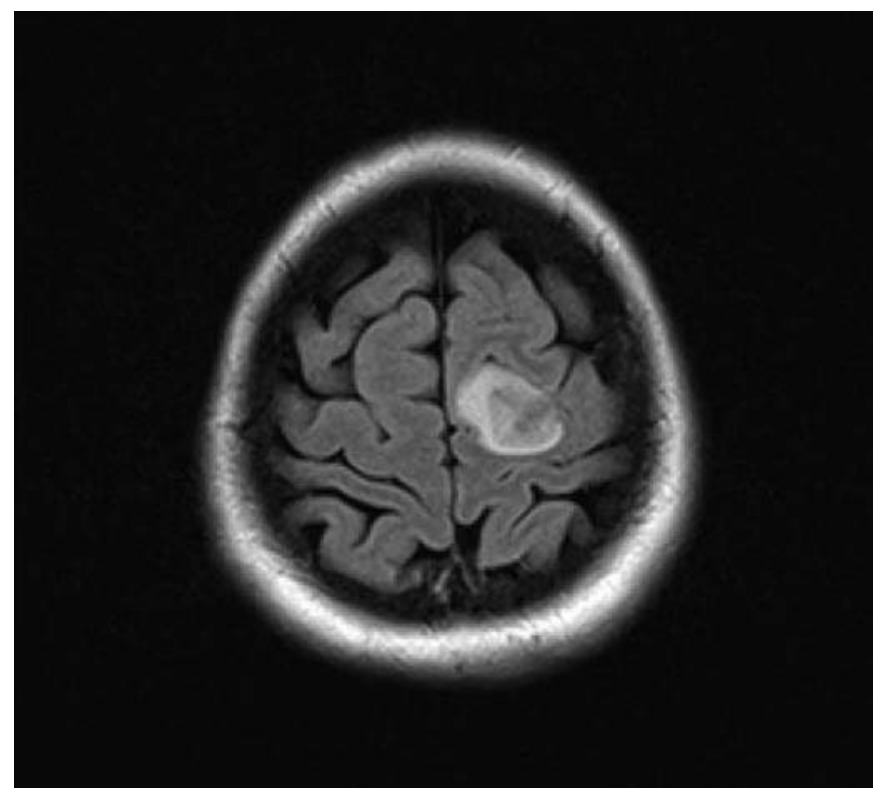

Figure 1: Axial FLAIR MRI showing glioma on initial diagnosis.

Steroids were started but there was no improvement in the patient's leg weakness. Our considerations during this time were supplementary motor cortex syndrome versus conversion disorder.

Upon probing further into the patient's history, it was found that she is married with a three-year-old son. She is a high school graduate and is unemployed. She has no previous medical illnesses and denies smoking, drinking, and recreational drug use. Her cultural roots are Guyanese from Indian descendants, and she married into a traditional Indian family. Her mother-inlaw lives with the family and the patient herself had little

From the Division of Neurosurgery (KJK, MB), Department of Psychiatry (SA), Toronto Western Hospita1, University Health Network, University of Toronto, Toronto, Canada.

Received August 13, 2009. Final Revisions Submitted September 28, 2009. Correspondence to: Mark Bernstein, Division of Neurosurgery, University of Toronto, Toronto Western Hospital, 399 Bathurst Street, 4West Wing, Toronto, Ontario, M5T 2S8, Canada 


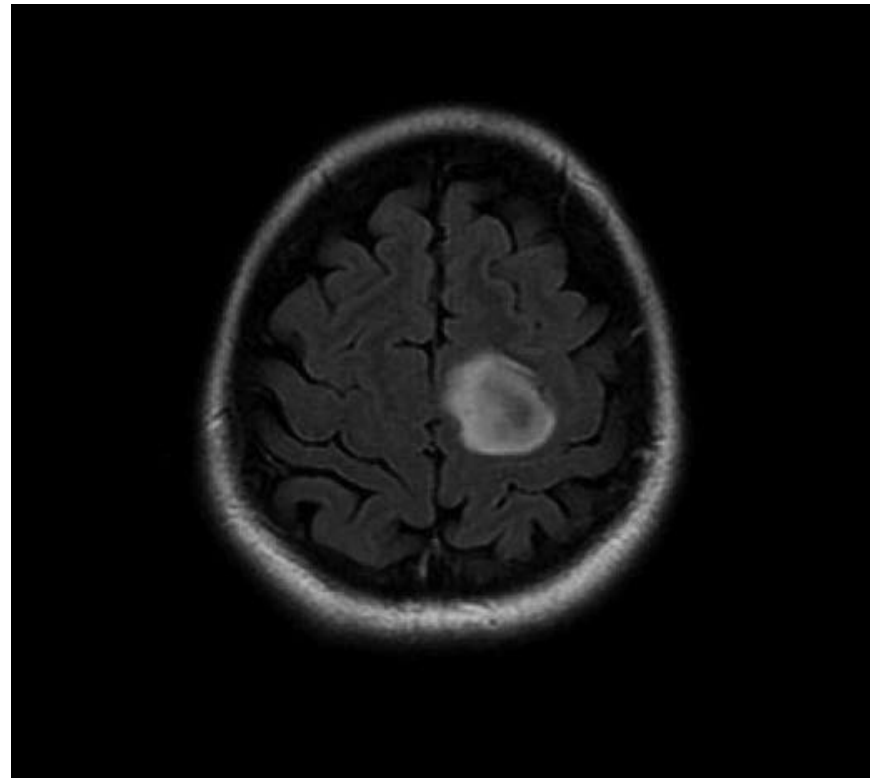

Figure 2: Axial FLAIR MRI showing glioma two years later, having grown.

authority in her own house. The patient suffered from a bout of depression a few months ago, wherein she attempted to commit suicide. She received psychiatric treatment and improved after a few weeks. On questioning, she admitted that the diagnosis of a brain tumour in addition to her unhappy domestic situation were the stressors that led her to attempt taking her own life.

In view of the inability to attribute the patient's symptoms to medical reasons despite extensive work-up, and the history of depression and presence of recent stressors, conversion disorder was highly considered. A psychiatric evaluation was obtained which confirmed that her condition was consistent with this diagnosis. The patient underwent counselling and physiotherapy with marked improvement in her symptoms. Five days after the surgery a flicker of movement returned to the patient's right leg, and two weeks after, there was anti-gravity movement in her proximal muscle groups. She was able to walk with support and perform her activities of daily living with assistance. She was then transferred to a rehab facility for further physiotherapy. Review five weeks post-operatively revealed almost full recovery and review two weeks after that revealed $100 \%$ recovery. At her most recent psychiatric follow-up, seven weeks after surgery, the patient was noted to have fully recovered and ambulated independently, although with slight caution. She continues to receive the current usual treatment for conversion disorder which involves psychoeducation, promoting acceptance of the psychiatric etiology, and managing the psychiatric issues that arose and that were postulated to lead to the development of her conversion monoplegia. The final histology of the tumour was anaplastic astrocytoma and the patient was referred to a neuro-oncologist for adjuvant treatment.

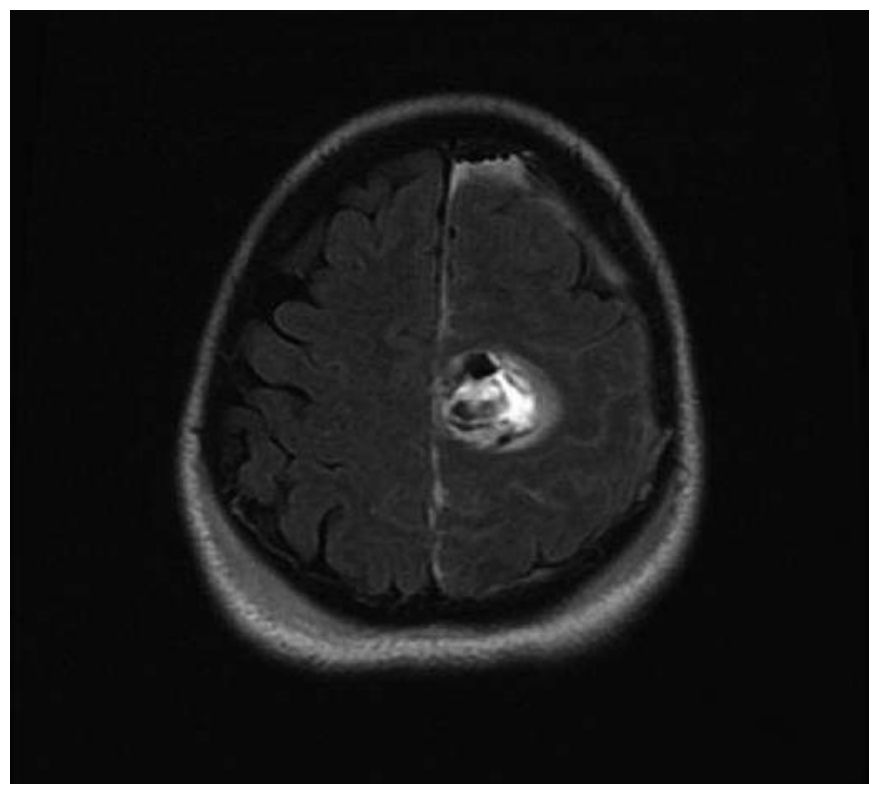

Figure 3: Axial FLAIR MRI 40 hours after surgery. There is no increase in edema or hemorrhage and probably residual tumour laterally.

\section{Discussion}

Conversion disorder is defined by the Diagnostic and Statistical Manual of Mental Disorders, fourth edition (DSMIV) ${ }^{1}$ as a mental disorder whose central feature is the appearance of symptoms affecting the patient's senses or voluntary movements that suggest a neurological or general medical disease or condition. These physical symptoms cannot be explained by an organic condition and seem to stem from psychological issues or conflicts. According to the DSM-IV criteria, conversion disorder is characterized by: (1) one or more symptoms affecting voluntary motor or sensory function; (2) resemblance to neurological or medical disease; (3) involvement of psychological factors; and (4) unintentional, unfeigned symptoms. ${ }^{2}$

The prevalence of conversion disorder is estimated to be between 11 and 300 per 100,000 people in the United States. ${ }^{1}$ Symptoms may begin at any age but usually occur during adolescence or early adulthood, and are seen more frequently in women than in men. ${ }^{1}$ Conversion disorder may also be more common in people with less formal education and those from minority ethnic groups, rural areas, and lower socioeconomic strata. ${ }^{2,3}$ Risk factors include female sex, lower educational level, physical or sexual abuse, emotional trauma, pre-existing psychopathology, and history of serious medical illness. ${ }^{4-6}$

The etiology for conversion disorder is unclear, but is presumed to be caused by severe stress, emotional conflict, or an associated psychiatric disorder. The mechanism is associated with two psychological concepts: primary gain and secondary gain. ${ }^{2}$ Primary gain refers to the lessening of anxiety by converting it into somatic symptomatology, thereby finding an outlet for the anxiety and protecting the patient from 
experiencing painful underlying feelings. ${ }^{3}$ This gives rise to $l a$ belle indifference, a condition wherein the patient seems surprisingly unconcerned about his or her physical symptoms. ${ }^{2}$ Secondary gain, on the other hand, is the subsequent benefit that the patient may derive from being in the sick role, such as avoidance of unpleasant tasks, removal from an uncomfortable situation, and increased attention, concern, and support from others. $^{2}$

Neurobiological correlates of conversion hemiplegia implicate frontal inhibition, likely influenced by emotional state from limbic and paralimbic areas, of the supplementary motor and motor areas. ${ }^{7}$ The anterior cingulate cortex, for example, with its role in linking emotional state and perception, was found to activate the frontal cortex leading to central inhibition of motor movement in a positron emission tomogram (PET) study of a patient with hemiparesis. ${ }^{8}$ Several studies observe that leftsided hemiplegia is more often noted in conversion disorder, suggesting some degree of anosognosia to explain the finding of la belle indifference. ${ }^{9}$

Diagnosis of conversion disorder involves the exclusion of organic neurological disease, the exclusion of malingering or intentional production of symptoms, and a history which supports a psychological mechanism. The evidence points to a chronic inadequate family environment and a history of abuse as the two major psychosocial factors. ${ }^{10}$

Treatment is generally supportive with explanation, reassurance, and physical therapy..$^{11}$ Behaviour therapy, cognitive therapy, and psychoeducation are recommended with the aim of promoting acceptance of the psychiatric etiology. ${ }^{12}$

It has been observed that patients with neurological problems have an increased incidence of conversion disorders, which may be explained by the theory that cerebral organic syndromes may change functioning of the brain in such a way as to provoke neurotic attitudes. ${ }^{2,4}$ Patients with structural brain lesions such as tumour, stroke, multiple sclerosis, and trauma, ${ }^{4,5,13,14}$ as well as those with neurophysiological disorders such as epilepsy ${ }^{4,15}$ have an increased prevalence of conversion disorder.

There are very few reports of conversion disorders in postoperative neurosurgical patients. Two case series described the occurrence of psychogenic seizures after brain surgery, usually for epilepsy.,16 There have also been reports of conversion disorder after implantation of a spinal cord stimulator for pain..$^{3,11}$ To our knowledge, there is no reported case in the literature of conversion disorder in an operated brain tumour patient manifesting as transient monoplegia.

Our patient fits the profile of an individual who is at high risk for conversion disorder: female, young adult, not highly educated, member of a cultural minority with a dysfunctional home environment, history of depression and suicidal ideation, history of a neurological condition (seizures secondary to a brain tumour), and lastly, the presence of a major life stress in the form of surgery for a malignant brain tumour. She had already been suffering from anxiety due to her medical condition and unhappy home life, and the additional stress from the surgery may have led her to develop conversion disorder. The tumour was located anterior to the motor cortex, and intraoperative cortical mapping ensured that this area was identified and spared during surgery. We acknowledge that we cannot be $100 \%$ certain that this is a conversion reaction and not an unusual variant of supplementary motor cortex syndrome, but the patient's constellation of neurologic signs and symptoms were not consistent with a neurological syndrome, and her rapid recovery did not follow the usual timeline for lesions in the motor cortex. In addition, she exhibited an unusual lack of concern about her symptoms and their implications. A battery of imaging and neurophysiologic tests failed to disclose the presence of structural or organic pathology that could explain the patient's symptoms. All of these led us to conclude that her transient post-op weakness was a manifestation of conversion disorder, which is a rare sequela after craniotomy for brain tumour resection.

\section{REFERENCES}

1. American Psychiatric Association. Diagnostic and statistical manual of mental disorders. 4th ed. Washington, DC: American Psychiatric Association; 1994. p. 457.

2. Owens C, Dein S. Conversion disorder: the modern hysteria. Advan Psychiatr Treat. 2006;12(2):152-7.

3. Parisod E, Murray RF, Cousins MJ. Conversion disorder after implant of a spinal cord stimulator in a patient with a complex regional pain syndrome. Anesth Analg. 2003;96(1):201-6.

4. Devinsky O, Mesad S, Alper K. Nondominant hemisphere lesions and conversion nonepileptic seizures. J Neuropsychiatry Clin Neurosci. 2001;13(3):367-73.

5. Babin PR, Gross P. Traumatic brain injury when symptoms don't add up: conversion and malingering in the rehabilitation setting. J Rehabil. 2002;68(2):4-13.

6. Binzer M, Andersen PM, Kullgren G. Clinical characteristics of patients with motor disability due to conversion disorder: a prospective control group study. J Neurol Neurosurg Psychiatry. 1997;63(1):83-8.

7. Aybek S, Kanaan RA, David AS. The neuropsychiatry of conversion disorder. Curr Opin Psychiatr. 2008;21(3):275-80.

8. Marshall JC, Halligan PW, Fink GR, Wade DT, Frackowiak RS. The functional anatomy of a hysterical paralysis. Cognition. 1997;64:B1-8

9. Black DN, Seritan AL, Taber KH, Hurley RA. Conversion hysteria: lessons from functional imaging. J Neuropsychiatry Clin Neurosci. 2004;16(3):245-51.

10. Reuber M, Howlett S, Khan A, Grunewald RA. Non-epileptic seizures and other functional neurological symptoms: predisposing, precipitating, and perpetuating factors. Psychosomatics. 2007;48(3):230-8.

11. Han D, Connelly NR, Weintraub A, Kanev P, Solis E. Conversion locked-in syndrome after implantation of a spinal cord stimulator. Anesth Analg. 2007;104(1):163-5.

12. Brooks JL, Goodfellow L, Bodde NM, Aldenkamp A, Baker GA. Nondrug treatments for psychogenic nonepileptic seizures: what's the evidence? Epilepsy Behav. 2007;11(3):367-77.

13. Caplan LR, Nadelson T. Multiple sclerosis and hysteria: lessons learned from their association. JAMA. 1980;243(23):2418-21.

14. Eames P. Hysteria following brain injury. J Neurol Neurosurg Psychiatry. 1992;55(11):1046-53.

15. Barry E, Krumholz A, Bergey GK, Chatha H, Alemayehu S, Grattan L. Nonepileptic posttraumatic seizures. Epilepsia. 1998;39(4): 427-31.

16. Ney GC, Barr WB, Napolitano C, Decker R, Schaul N. New-onset psychogenic seizures after surgery for epilepsy. Arch Neurol. 1998;55(5):726-30. 